\title{
VALIDATION OF WELDING SIMULATIONS USING THERMAL STRAINS MEASURED WITH DIC
}

\author{
Maarten De Strycker ${ }^{1, a}$, Pascal Lava ${ }^{1, b}$, Wim Van Paepegem ${ }^{2, c}$, Luc \\ Schueremans $^{3, d}$ and Dimitri Debruyne ${ }^{4, e}$ \\ ${ }^{1}$ MeM2P research group, Catholic University College Ghent \\ Gebroeders Desmetstraat 1, 9000 Ghent, Belgium \\ ${ }^{2}$ Department of Materials Science and Engineering, Ghent University \\ St.-Pietersnieuwstraat 41, 9000 Ghent, Belgium \\ ${ }^{3}$ Department of Civil Engineering, KULeuven \\ Kasteelpark Arenberg 40, 3001 Heverlee, Belgium \\ ${ }^{4}$ Department MTM, KULeuven \\ Kasteelpark Arenberg 44, 3001 Heverlee, Belgium
}

${ }^{a}$ maarten.destrycker@kahosl.be, ${ }^{b}$ pascal.lava@kahosl.be, ${ }^{c}$ wim.vanpaepegem@ugent.be,
${ }^{d}$ luc.schueremans@bwk.kuleuven.be, ${ }^{e}$ dimitri.debruyne@mtm.kuleuven.be

Keywords: DIC, strain gauge, tube, welding, stainless steel

\begin{abstract}
Residual stresses can affect the performance of steel tubes in many ways and as a result their magnitude and distribution is of particular interest to many applications. Residual stresses in cold-rolled steel tubes mainly originate from the rolling of a flat plate into a circular cross section (involving plastic deformations) and the weld bead that closes the cross section (involving nonuniform heating and cooling). Focus in this contribution is on the longitudinal weld bead that closes the cross section. To reveal the residual stresses in the tubes under consideration, a finite element analysis (FEA) of the welding step in the production process is made. The FEA of the welding process is validated with the temperature evolution of the thermal simulation and the strain evolution for the mechanical part of the analysis. Several methods for measuring the strain evolution are available and in this contribution we investigate if the Digital Image Correlation (DIC) technique can record the strain evolution during welding. It is shown that the strain evolution obtained with DIC is in agreement with that found by electrical strain gauges. The results of these experimental measuring methods are compared with numerical results from a FEA of the welding process.
\end{abstract}

\section{Introduction}

Residual stresses affect the application of steel tubes in many ways and their magnitude and distribution are of particular interest. Residual stresses in cold-rolled steel tubes are inherent to the production process: first a flat steel plate is rolled into a circular cross-section and then both edges of the plate are welded together to obtain a closed cross-section. The first step involves nonhomogeneous plastic deformations and the second imposes a non-uniform heating and cooling cycle. The focus in this research is on the residual stresses due to the welding step.

To reveal the residual stresses in the tubes under consideration, a finite element analysis (FEA) is made. FEA has the advantage that all the residual stress components after welding in the whole tube are revealed. The result of the simulation is however very dependent on the input parameters: thermo-mechanical temperature-dependent material properties, thermal load definition, thermal and mechanical boundary conditions. Therefore, validation of the FEA results is necessary. 
Welding simulations are usually split up into two separate analyses: first the temperature evolution in the welded construction is calculated from the heat input and next, starting from the temperature field, the stress and strain development due to the temperature cycle is calculated in a mechanical analysis. The thermal field used, for the mechanical simulation, can be validated by measuring the transient temperatures in the construction. The mechanical analysis, in which more material parameters are introduced and more assumptions are made, induce a more complicated stress-strain validation.

Lindgren [1] gives an extensive overview of papers describing validation methods for the mechanical analysis. These include residual strain measurements using hole-drilling techniques, Xray techniques, or neutron diffraction measurements; transient strains have also been measured in some cases using high-temperature strain gauges. Other sources state strain evolution measurement during a welding operation with a laser speckle method [2] and measuring distortions with displacement sensors [3,4]. Residual stress measurements can be done with synchrotron X-ray diffraction [5,6] and the hole drilling method [7].

The mentioned methods have some drawbacks: only in a restricted number of points the deformations and strains are available (in the case of displacement sensors and strain gauges), temperature compensation is needed (for strain gauges), only the final stress-strain state is known (for residual stress measurements), the part is lost (destructive residual stress measuring methods), the method requires a complex setup (laser interferometry, X-ray diffraction and neutron diffraction residual stress measurements), is sensitive to vibrations, differences in the density of the air between the measurement device and the welded object (laser interferometry).

An alternative method which counters some of these drawbacks is the Digital Image Correlation (DIC) technique. Compared to other measuring methods used in welded structures, DIC has the major advantages that it is a contactless method; that it can uncover the strain evolution over a significant portion of the surface when a work piece is welded, even in zones that heat up to $600^{\circ} \mathrm{C}$ and without the need for thermal compensation on the measured strains. It has, however, the drawback that it is conventionally not judged suited for small strains (below $1000 \mu \mathrm{m} / \mathrm{m}$ ) that occur in the welding case under consideration here. In this contribution it is investigated whether it is possible to observe, with sufficient accuracy, the deformations during a welding process, using DIC. Thermal strains up to $650^{\circ} \mathrm{C}$ were already successfully measured with DIC on uniformly heated 3D tubular specimens by the authors [8], under the conditions that sufficient data are collected by taking enough images, that the strain is taken as the average over the homogeneous strain area, and putting the temperature limit to $600^{\circ} \mathrm{C}$.

As it is the first time that the DIC technique is used for measuring inhomogeneous thermal strain fields in the range below $1000 \mu \mathrm{m} / \mathrm{m}$, the strain field measured with the DIC technique is compared with the strains obtained by electrical strain gauges, attached to the tube as a reference.

\section{Experimental work}

Setup. The experimental setup is a modified version of an actual industrial welding setup. In the laboratory, the tube is kept static and the welding torch is moved on top of it by a robot. To avoid overexposure on the images taken during the welding, the blazing light of the GTAW weld beam is shielded by a tin foil wrapped around the cup of the torch. The use of a robot assures controlled welding conditions. Only a confined length of the tube $(300 \mathrm{~mm})$ is welded. To avoid complications of applying closing forces (which would hamper the making of the images for DIC), the welding experiments starts from an already finished tube, i.e. a tube that was rolled and welded before.

The tubes under consideration are thin-walled tubes (typical diameter 55 to $60 \mathrm{~mm}$, thickness between 1.2 and $2 \mathrm{~mm}$ ) made of stainless steel with a length of $400 \mathrm{~mm}$.

The tubes are equipped with a speckle pattern on one side and two strain gauges and five thermocouples on the opposite side, see Fig. 1. Thermocouples are used to validate the thermal field of the simulations (see section "Finite element simulation") and to calculate the thermal strain 
measured with the strain gauges. Results are given in Fig.4(a). To know the temperature in the material under the strain gauge, the thermocouples are placed around and close to the strain gauge.

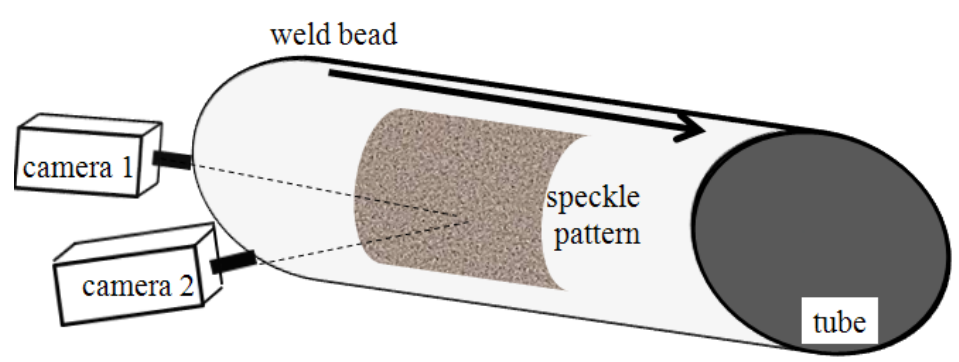

(a)

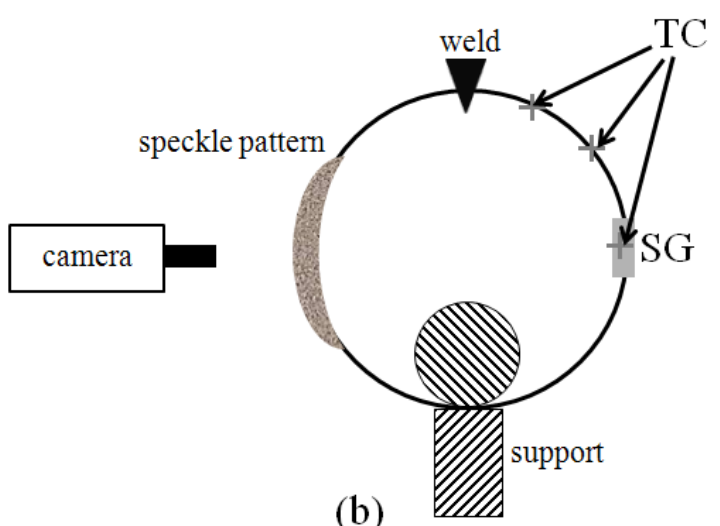

(b)

Fig. 1: sketch of the setup of the tube with the DIC cameras and indication of the strain gauge (SG) and thermocouple (TC) position. Thermocouples are positioned around the strain gauges.

Strain gauge measurements. As can be seen from Fig. 1, the position of the strain gauges is symmetrical to the middle of the speckle pattern. The strain gauges should thus measure the same strain during the welding as found in a corresponding area in the middle of the speckle pattern. As the steel under the strain gauge heats during the welding, spurious thermal strains need to be compensated. Strain gauge results are thus depending on the accuracy of the temperature measurement. TC6 (see Fig. 4(a)) is in between the two strain gauges. This curve gives an indication of the temperature cycle that the strain gauge is subjected to during the welding.

DIC measurements. At one side of the tube, in an area of about $40 \mathrm{~mm}$ by $200 \mathrm{~mm}$, a uniform white paint layer is put on the tube. On this white paint, a black random speckle pattern is applied with a maximum speckle size of about $1 \mathrm{~mm}^{2}$. Markers (black crosses) are integrated in this speckle pattern as a reference for the focus of the cameras, see Fig.2. These markers are also used in the processing of the images for assigning a coordinate system and for defining the position where the strain is extracted.

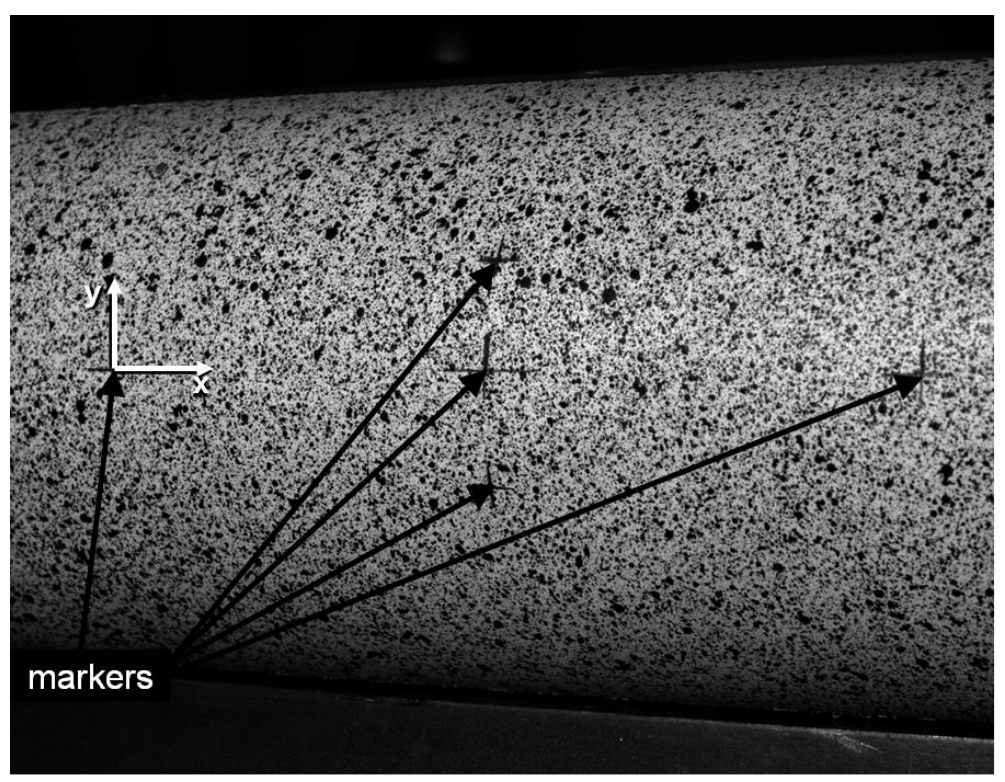

Fig. 2: Typical speckle pattern for this setup. Black crosses are used as reference markers for the definition of the coordinate system: the $\mathrm{x}$-axis is in longitudinal direction of the tube, the y-direction is in hoop direction. 
2400 images are taken to follow the strain evolution in the tube during the welding: in the first 600 s two images per second and in the following 1200s one image per second.

The calculation of the DIC displacements and strains was performed with the inhouse developed DIC software platform MatchID. In view of the smooth deformations in the application under investigation, the subset deformation is restricted to an affine transformation during the analysis. As the displacements from one image to the other may be smaller than one pixel, the subset in the deformed image is not likely to fit on the pixel grid and an interpolation method between the pixels is needed. Previous research $[9,10]$ has shown that the approximate normalised sum of squared difference (ANSSD) correlation algorithm with cubic B-spline interpolation reduces the systematic errors in the correlation process. In this way, for every pixel in the undeformed image, the displacement in all the deformed images can be calculated. In practice a certain step size is applied, i.e. for a step size of $3 \mathrm{px}$, the subset is moved 3px from its original position, which means that displacement results are not available for every pixel, but on a grid three times coarser than the pixel grid. In order to avoid cumulative errors in this small deformation application, it is appropriate to use always the first image as the reference image.

Next, the obtained displacements are smoothed via a so-called strain window (SW) method to reduce the impact of uncertainties and noise. This is a commonly adopted technique during the process of strain derivation [11]. In this step of the analysis, a plane is fit through the displacement values in the points around the centre of the strain window (CSW) where displacements are available. This analytical approximation makes the calculation of the full-field strain information straightforward. In this research a default strain window of 5 is used. This means that the displacement field is smoothed over an area of $5 \times 5$ displacement data points, or with a step size of 3px, over an area of $12 \times 12$ px around the CSW. A full-field strain map is shown in Fig. 3.

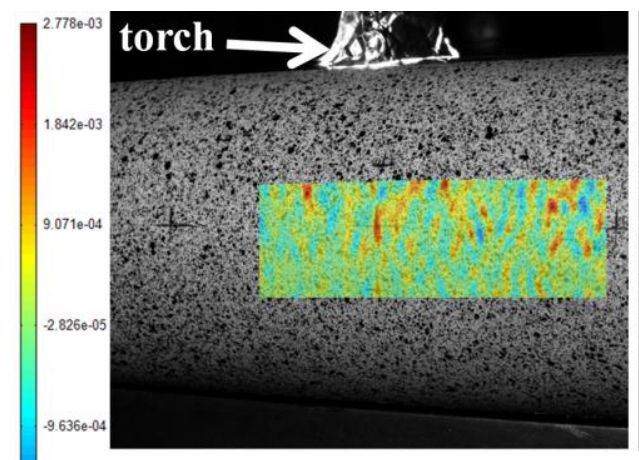

(a) $\varepsilon_{\mathrm{xx}}$

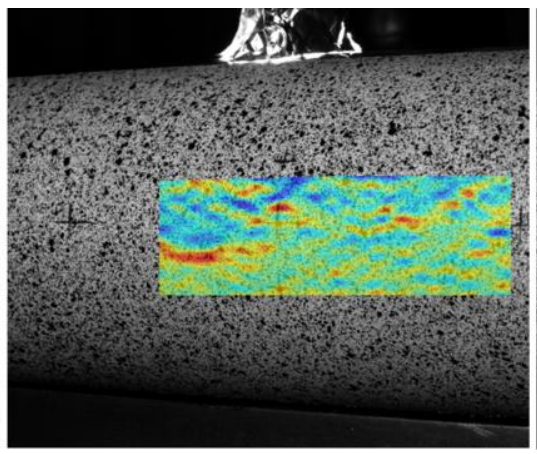

(b) $\varepsilon_{\mathrm{yy}}$

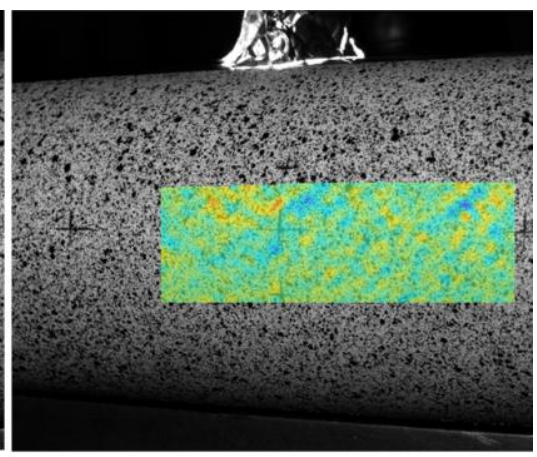

(c) $\varepsilon_{\mathrm{xy}}$

Fig. 3: full field strain map of the strain during the welding at $t=75 \mathrm{~s}$ in (a) longitudinal and (b) hoop direction; (c) shear strain. The torch is shielded by a tin foil.

As one pixel has a dimension of about $0.072 \mathrm{~mm}$ in the current images and the measuring grid of the strain gauge is $3 \times 6 \mathrm{~mm}^{2}$, the strain obtained from a strain gauge is in fact the average strain over this area, or speaking in terms of DIC, the strain averaged over a surface area of 40px $\times 80 \mathrm{px}$. To compare the strains obtained with DIC and those with a strain gauge, strains of all the CSW within the area of a strain gauge were averaged and taken as the strain to compare with the strain gauge results. This means that the strains plotted in Fig. 4 are averaged over a region of $40 \mathrm{px} \times$ 80px, corresponding to $355 \mathrm{CSW}$ (as the step size in this example is $3 \mathrm{px}$ ).

The resulting strain evolution during the welding and the cooling of the tube in an area corresponding to the strain gauge in the hoop direction is plotted in Fig.4(b). For the strain gauge in longitudinal direction, similar results are found. 


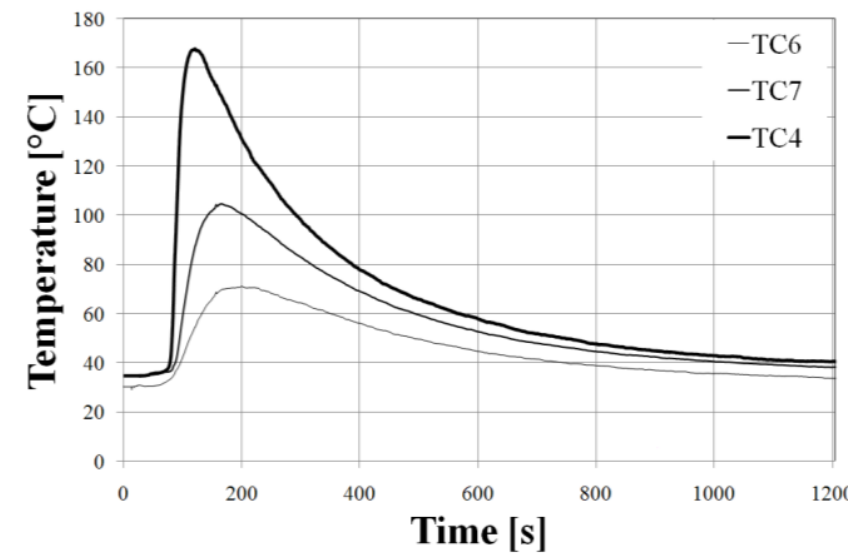

(a)

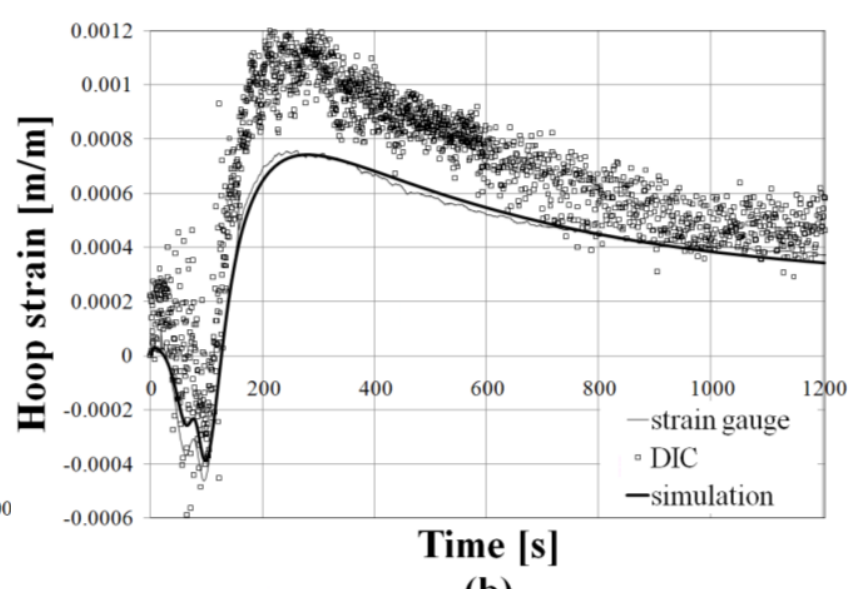

(b)

Fig. 4: Experimentally obtained results: selection of temperature measurements for different thermocouples (TC) (a) and (b) strains in hoop direction compared to the simulated strain curve.

\section{Finite element simulation}

The welding process is numerically simulated with Abaqus. As is usual in welding simulation, the analysis is split up into two simulations, first the temperature field is calculated and then, based on the calculated temperatures, the thermal strain field is calculated.

In the thermal simulation, the parameters with the most influence on the result are: the heatinput, the convection coefficient and the emissivity. The emissivity was measured experimentally and the other two parameters were defined with a Levenberg-Marquardt optimization method which minimizes the difference between the temperature curves measured with the thermocouples in the experiment and those calculated with the FEA. This procedure yields a simulated temperature field that is in agreement with the measured temperature field in the tube.

Then the mechanical analysis, which calculates the stress-strain development in the tube during the welding, is made. The strain evolution in different points can be followed to validate the model (see Fig. 4) and at the end of the simulation the residual stress pattern in the tube is known. An important difference with the tube in the experiments is that the tube in the simulation is initially stress free. This is not the case in the experimental tube as the tube was already rolled and welded before.

\section{Discussion of the results}

Comparison strain gauges and DIC. From Fig. 4(b) it is clear that the strain gauge curves do not completely coincide with the DIC curves. As was indicated already in the introduction, due to the small deformations in the actual setup, a large scatter could be expected on the DIC results. This makes it difficult to distinguish the small strain evolution in the first 150 s of the experiment. Moreover, the DIC results are not averaged in time, which is the case for the strain gauge results. Still, it is possible to recognize the same strain evolution in the DIC measurement as in the strain gauge measurement and strains are of the same order of magnitude. Important to remark is that the DIC results are not dependent on the temperature measurement. Indeed, the temperatures are measured with thermocouples and these give the surface temperature of the material. However, a bad contact between the material and the thermocouple measuring point yields a lower read-out temperature than the actual material temperature. Care should be taken when applying the measured temperatures for temperature compensation of the strain gauge measurements.

Comparison experimental-simulation. Although it might be difficult to distinguish, the experimental and the numerical curves show a similar strain evolution. Again, the strains found are in the same order of magnitude. 
The match of the strain evolution between the strain gauge and the FEA for the hoop strain shows clearly the dependency on the temperature measurement: both are based on the same temperature curves and might thus both have the same error.

If the DIC results are taken as the reference for the FEA validation, the temperature measurement error is not present. It is obvious that having results over a certain area of the tube will allow a better validation compared to the results only available from a limited number of points.

Further it should be mentioned that averaging the DIC results over several pixels is necessary as the noise on the data is higher the lesser pixels are taken into account for the strain calculation. This however follows naturally as the finite elements in the simulation always coincide with an area representing a number of pixels. It should be noted that more experimental data will allow to define a confidence interval of the found results.

\section{Conclusion and future work}

The results presented here show that DIC is a viable method for validating the FEA results of a welding simulation. The differences between the strain gauge result and the DIC strain curves are due to the temperature compensation, which requires accurate temperature measurements. This fact again shows the complexity of strain gauge measurements in a welding setup. Future work will focus on more reliable temperature measurement techniques in the experimental setup.

The differences between the numerical and the experimental results can be attributed to the initial stress field in the experimental tubes, the dependence of the stress-strain evolution on the thermal input of the simulation and uncertainties in the input parameters for the mechanical analysis. Future work will thus consist of obtaining stress free tubes for the experimental part of the research and obtaining the residual stress field due to rolling for the numerical part of the research.

\section{References}

[1] L. Lindgren, Journal of Thermal Stresses, 2001, 24, 305-334.

[2] Y. Muramatsu, S. Kuroda and C. Shiga, Welding International, 2003, 17(8), 615-623.

[3] Y. Mikami, M. Mochizuki and M. Toyoda: 'Mathematical modelling of weld phenomena 8', eds. H. Cerjak, H. Bhadeshia and E. Kozeschnik, Technische Universität Graz, 981-1001.

[4] C. Schwenk, M. Rethmeier and D. Weiss: 'Mathematical modelling of weld phenomena 8', eds. H. Cerjak, H. Bhadeshia and E. Kozeschnik, Technische Universität Graz, 835-846.

[5] E. Van der Aa, R. Thiessen, A. Murugaiyan, M. Hermans, J. Sietsma and I. Richardson: 'Mathematical modelling of weld phenomena 8', eds. H. Cerjak, H. Bhadeshia and E. Kozeschnik, Technische Universität Graz, 387-408.

[6] E. Van der Aa, M. Hermans and I. Richardson: 'Mathematical modelling of weld phenomena 8', eds. H. Cerjak, H. Bhadeshia and E. Kozeschnik, Technische Universität Graz, 1053-1072.

[7] S. Courtin and P. Gilles: 'Mathematical modelling of weld phenomena 8', eds. H. Cerjak, H. Bhadeshia and E. Kozeschnik, Technische Universität Graz, 603-626.

[8] M. De Strycker, L. Schueremans, W. Van Paepegem and D. Debruyne, Optics and Lasers in Engineering, 2010, 48, 978-986.

[9] P. Lava, S. Cooreman, S. Coppieters, M. De Strycker and D. Debruyne, Optics and Lasers in Engineering, 2009, 47, 747-753.

[10]H. Schreier, J. Braasch and M. Sutton, Optical Engineering, 2000, 39, 2915-2921.

[11]P. Lava, S. Cooreman and D. Debruyne, Optics and Lasers in Engineering, 2010, 48, 457-468. 